\title{
Anticoagulação durante gravidez de mulher portadora de cardiopatia
}

\author{
Anticoagulation during pregnancy of woman with heart diseases
}

\section{Walkiria Samuel Ávila*}

\section{Resumo}

Diretrizes em muito contribuem para minimizar os potenciais riscos obstétricos e fetais e melhorar a assistência durante o ciclo gravídico-puerperal. Contudo, há controvérsias a respeito do manuseio da anticoagulação em mulheres portadoras de cardiopatias. A importância deste assunto assenta-se no fato de que o tromboembolismo é considerado uma das principais causas de morte materna em portadoras de cardiopatia, o que torna obrigatório o conhecimento sobre sua prevenção. A estratégia do tratamento antitrombótico é ponderada pelo risco de trombose imposto pela situação clínica materna e pelos efeitos adversos que os anticoagulantes podem causar ao concepto. Neste artigo, serão discutidas a estratificação do risco materno ao tromboembolismo, as propriedades dos anticoagulantes indicados para a sua prevenção e a estratégia terapêutica nos diversos momentos da gestação, parto e puerpério da mulher portadora de cardiopatia.

Palavras-chave: Tromboembolismo, anticoagulação, cardiopatia, gravidez.

\section{Introdução}

A anticoagulação durante a gravidez é uma situação que, habitualmente, suscita dilema na prática médica, uma vez que significa buscar a prevenção do tromboembolismo materno e a segurança, evitando-se a hemorragia e a teratogenicidade fetal.

\section{Risco de tromboembolismo na gravidez}

O tromboembolismo é considerado uma das principais causas de morte durante a gravidez de mulheres portadoras de cardiopatias ${ }^{1}$. A doença valvar reumática, que prevalece como sendo a cardiopatia mais frequente na mulher jovem, favorece condições de risco à ocorrência de tromboembolismo na gestação, seja em valva natural como em prótese valvar.

Em estudo realizado com 19 casos de tromboembolismo na gestação, verificou-se que a doença valvar reumáti-

\begin{abstract}
Guidelines provide great contribution to the reduction of potential obstetric and fetal risks and to the improvement of health care during pregnancy and puerperium. However, there are still controversies regarding the management of anticoagulation in women with heart diseases. The importance of this issue is based on the fact that thromboembolism is considered one of the main causes of maternal death in women with heart diseases; therefore, the acquisition of knowledge about how to prevent this disorder is mandatory. The strategy of the antithrombotic treatment is based on the risk of thrombosis imposed by the maternal clinical picture and on the adverse effects of the anticoagulants for the conceptus. In the present study, we discussed the stratification of maternal risk of thromboembolism, the properties of the anticoagulants indicated for its prevention, and the therapeutic strategy at different moments of pregnancy, delivery and puerperium of women with heart diseases.
\end{abstract}

Keywords: Thromboembolism, anticoagulation, heart disease, pregnancy.

ca correspondeu a $12(63,1 \%)$ e a seis $(31,6 \%)$ casos distribuídos em cardiopatia congênita e hipertensão arterial pulmonar, respectivamente ${ }^{1}$.

O potencial tromboembólico está centrado, habitualmente, na situação clínica da paciente, mas é agravado pelas particularidades inerentes à gravidez, como as reológicas, mecânicas e hormonais (Tabela 1), mais proeminentes a partir do segundo trimestre da gravidez e culminando no puerpério. Somam-se a elas outros fatores como idade, paridade, tabagismo, obesidade, repouso prolongado, parto cesárea, que elevam a incidência de fenômeno tromboembólico no ciclo gravídico-puerperal.

As diretrizes em anticoagulação durante a gravidez estratificam as cardiopatias em riscos de trombose (Tabela 2), nos quais devem ser ponderados o benefício da anticoagulação e o prejuízo obstétrico e fetal. As situações de alto risco exigem a anticoagulação permanente, en-

\footnotetext{
* Professora livre-docente em Cardiologia. Faculdade de Medicina da Universidade de São Paulo (FMUSP). Médica-chefe, Setor de Cardiopatia e Gravidez do Instituto do Coração (InCor). Hospital das Clínicas da FMUSP.

Não foram declarados conflitos de interesse associados à publicação deste artigo.

Artigo submetido em 09.02.09, aceito em 17.12.09.

J Vasc Bras. 2010;9(1):42-45.

Copyright @ 2010 by Sociedade Brasileira de Angiologia e de Cirurgia Vascular
} 
Tabela 1 - Fatores da gravidez que predispõem ao tromboembolismo

\begin{tabular}{llll}
\hline Reológico & Mecânico & Hormonal & Outros \\
\hline Aumento na concentração de: & Redução do retorno venoso & Modificação da complacência arterial & Parto cesárea \\
- Fatores V, VI, VII, IX e X & & Obesidade \\
- Atividade plaquetária & & Repouso prolongado \\
- Síntese de fibrinogênio & & Pré-eclampsia \\
& & & Infecções \\
\hline
\end{tabular}

quanto nas de menor risco ela pode ser transitória ou controversa $^{2}$.

O tromboembolismo e as complicações hemorrágicas constituem as maiores desvantagens das próteses mecânicas e correspondem a 20 e $2,5 \%$ dos casos, respectivamente. No período de 2000 a 2006 foram estudadas no Instituto do Coração (InCor) 650 gestantes com cardiopatias, dentre as quais $64(9,8 \%)$ utilizaram anticoagulantes. A valvopatia reumática correspondeu a 50\% dessa casuística, incluindo pacientes com fibrilação atrial e próteses mecânicas; a cardiopatia congênita, a 14\% e a cardiomiopatia dilatada, aos restantes $12 \%$ das gestantes cardiopatas ${ }^{1}$.

\section{Propriedades dos anticoagulantes na gravidez}

A heparina não fracionada (HNF) e a heparina de baixo peso molecular (HBPM) não atravessam a barreira placentária e, por isso, são, conceitualmente, os anticoagulantes preferenciais durante a gravidez. Contudo, o seu uso prolongado associa-se a efeitos colaterais maternos, incluindo trombocitopenia, hemorragia e osteoporose. Somam-se a isso o desconforto da aplicação, as dificuldades de controle da anticoagulação e o custo.

Tabela 2 - Principais indicações cardíacas de anticoagulação na gravidez $^{2}$

\begin{tabular}{lll}
\hline Permanente & Alto risco & Prótese valvar mecânica \\
\hline \multirow{2}{*}{ Transitória } & Menor risco & Fibrilação atrial permanente \\
& Antecedente tromboembólico \\
& & Trombose venosa profunda: \\
& tratamento e prevenção \\
& Cardioversão elétrica \\
& & Cardiomiopatia dilatada \\
& & Insuficiência cardíaca congestiva \\
& Cardiopatias congênitas cianóticas \\
& Trombo intracavitário \\
& Repouso prolongado
\end{tabular}

As vantagens da HBPM sobre a HNF são a superioridade quanto à biodisponibilidade (100 versus 30\%), à vida média após a aplicação ( $2 \mathrm{~h}$ versus $1 \mathrm{~h}$ ), à absorção por via subcutânea (100 versus variável) e à menor incidência de trombocitopenia ( 0 versus $2,7 \%$ ). No entanto, existem limitações ao seu uso que incluem a neutralidade inadequada pelo sulfato de protramina e o custo mensal ${ }^{3}$.

O uso permanente da varfarina está indicado em pacientes com próteses mecânicas e fibrilação atrial, porém apresenta riscos para o feto com graus diferentes a cada trimestre. A varfarina atravessa a barreira placentária, acarretando de 1 a $3 \%$ de malformações congênitas e caracterizando-se pela síndrome varfarínico-fetal, que ocorre entre a sexta e a nona semana de gestação e favorece ao abortamento espontâneo em cerca de 10 a 30\% dos casos. No segundo e terceiro trimestres, ela acarreta restrição de crescimento intrauterino, prematuridade, anomalia do sistema nervoso central e morte neonatal devido à hemorragia cerebral decorrente da compressão do polo cefálico no período expulsivo ${ }^{4}$.

\section{Recomendações para o uso de anticoagulantes na gravidez}

Não há consenso sobre qual seria a melhor anticoagulação para as gestantes portadoras de cardiopatias em face dos diversos riscos presumidos de trombose, porque nenhuma opção farmacológica isolada ou combinada forneceu evidências quanto à eficácia em não acarretar efeitos colaterais para a mãe e o feto.

Os estudos clínicos continuam apoiando o emprego da varfarina na anticoagulação permanente para portadora de próteses mecânicas e desencorajando o uso da HNF subcutânea. Dados do InCor reforçam os da literatura ao mostrarem que $85 \%$ dos casos de tromboembolismo durante a gravidez em portadoras de próteses mecânicas se associaram ao uso da HNF subcutânea ${ }^{5,6}$. 
A HBPM ganhou espaço na anticoagulação transitória com risco menor e está gradualmente tomando o lugar da HNF subcutânea também em casos de pacientes de alto risco, como nos casos de próteses mecânicas. Há uma tendência a indicar a HBPM em cardiopatias ou situações de menor risco à trombose, que incluem: fibrilação atrial permanente, hipertensão pulmonar, acidente vascular prévio e durante a cardioversão elétrica. Nessas situações, o tempo de uso do anticoagulante pode ser limitado ao terceiro trimestre e ao puerpério, momentos da hipercoagulabilidade.

Chan et al. ${ }^{7}$ revisaram 1.234 gestações de mulheres portadoras de próteses mecânicas e mostraram que a substituição da varfarina pela heparina no primeiro trimestre potencialmente elimina a embriopatia fetal, mas aumenta o risco do tromboembolismo.

As recomendações atuais do setor de Cardiopatia e Gravidez do InCor, de acordo com o risco de trombose, são as expostas a seguir.

\section{Situações de maior risco}

Opção 1: recomenda-se, na primeira semana de atraso menstrual e com beta gonadotrofina coriônica humana ( $\beta \mathrm{HCG}$ ) positiva, substituir a varfarina por heparina de baixo peso molecular - enoxaparina $1 \mathrm{mg} / \mathrm{kg} / \mathrm{dose}$ subcutânea a cada 12 horas até a $13^{\mathrm{a}}$ semana; reintroduzir a varfarina $\mathrm{VO}$ e mantê-la até a $36^{\mathrm{a}}$ semana, quando a paciente deve ser hospitalizada; substituir a varfarina pela heparina não fracionada na dose de 10 a $12 \mathrm{UI} / \mathrm{kg} / \mathrm{h}$ IV até 4 horas antes do parto. Seis horas depois do parto, reintroduzir a heparina IV nos mesmos moldes de administração do pré-parto e, 48 horas depois, a varfarina.

Opção 2: a paciente que iniciar o pré-natal após a sexta semana de gestação, ou após duas semanas de atraso menstrual, não deve interromper a varfarina até a $36^{\mathrm{a}}$ semana, seguindo a opção 1.

Opção 3: a paciente que inicia o pré-natal sem anticoagulante repete a opção 1 .

Os ajustes da dose devem ser realizados de acordo com o coagulograma, de forma que a dose de varfarina seja controlada pelo International Normalized Ratio (INR) entre 2,5 e 3,5 semanalmente e, posteriormente, quinzenalmente; a enoxaparina deve ser administrada mantendo-se o fator anti-Xa entre 0,6 e $0,8 \mathrm{UI} / \mathrm{mL}$, que deve ser colhido 4 horas após a aplicação, com controle semanal quando atingir o nível terapêutico; a dose da heparina não fracionada deve ser regulada pelo tempo de tromboplastina parcial ativada (TTPA) mantido entre 1,5 e 2,5 vezes o valor normal, com controle duas vezes ao dia até atingir o nível terapêutico e, posteriormente, uma vez ao dia. Deve-se acompanhar a contagem de plaquetas periodicamente devido ao risco de plaquetopenia.

A indicação do tipo de parto é obstétrica, porém, para as pacientes em uso de varfarina sódica, a opção é pelo parto cesáreo devido ao menor risco de hemorragia cerebral neonatal. Em casos de cesárea de urgência com INR alto, em uso de varfarina sódica, ajusta-se a INR com administração IV de plasma fresco $10 \mathrm{~mL} / \mathrm{kg}$ (duas a três bolsas) + Vitamina K (Kanakion MM 2,5 mg IM) ou, em substituição a esta última, complexo protrombínico $10 \mathrm{UI} / \mathrm{kg}$ diluído em $50 \mathrm{~mL}$ de solução fisiológica para infusão IV. A INR para a realização do parto cesáreo com segurança deve ser $\leq 1,5$; se a INR estiver entre 2,0 e 5,0, corrige-se com plasma fresco ou complexo protrombínico; e se for $>5,0$, administra-se plasma fresco ou complexo protrombínico associando-se vitamina $\mathrm{K}$. O uso da varfarina no puerpério não contraindica a amamentação.

\section{Situações de menor risco}

Opção 1: enoxaparina $40 \mathrm{mg} / \mathrm{dia}$ até a $13^{\mathrm{a}}$ semana; posteriormente, varfarina (mantendo a INR entre 2 e 3 ) até a $36^{\mathrm{a}}$ semana; retornar à enoxaparina em nível profilático até 24 horas antes do parto, manter sem medicação anticoagulante e, seis horas após a punção ou a retirada do cateter, reintroduzir a enoxaparina (que deve ser mantida até o ajuste adequado da INR); 48 horas depois recomeçar com a varfarina.

Opção 2: enoxaparina 40 mg/dia durante toda a gestação.

Opção 3: heparina não fracionada 10.000 U SC $12 / 12 \mathrm{~h}$ até a $13^{\mathrm{a}}$ semana e, após a $36^{\mathrm{a}}$ semana, $10.000 \mathrm{U}$ SC $8 / 8 \mathrm{~h}$.

\section{Planejamento familiar}

A orientação para o planejamento de futura gravidez e a anticoncepção exige o respeito à autonomia do paciente que faz uso permanente de anticoagulante oral. Deve-se, portanto, destacar que não há contraindicação à gestação, mas alertar para o potencial de morbidade do uso da varfarina sódica. De modo geral, há uma tendência a desmotivar 
prole com mais de dois filhos em função da morbiletalidade materna e fetal que incluem hemorragia, tromboembolismo, abortamento, teratogênese, às que se acrescentam os custos e a hospitalização prolongada. O diálogo franco com o casal é fundamental.

Nesse aspecto, deve-se ponderar a escolha da laqueadura tubária para casais com intenção de não terem mais filhos, ou métodos reversíveis de contracepção, para outras situações ${ }^{8}$. Dentre os anticoncepcionais que não provocam interação com anticoagulantes, destacam-se os compostos com progesterona, que incluem as formas injetáveis trimestral, por via oral e as implantáveis no tecido subcutâneo.

Em conclusão, o papel do cardiologista é o de conscientizar a paciente de que estar adequadamente anticoagulada representa uma condição essencial para o desenvolvimento da gravidez, porém não a isenta de riscos para si e para o seu filho.

\section{Referências}

1. Ávila WS, Rossi EG, Ramires JAF, et al. Pregnancy in patients with heart disease: experience with 1,000 cases. Clin Cardiol. 2003;26:135-42.
2. Ávila WS, Grinberg M. Anticoagulação, gravidez e cardiopatia. Uma tríade, três domínios e cinco momentos. Arq Bras Cardiol. 2005;84:44-8.

3. Hirsh J, Raschke R. Heparin and low-molecular-weight heparin: the Seventh ACCP Conference on Antithrombotic and Thrombolytic Therapy. Chest. 2004;126(3 Suppl):188S-203S.

4. Cotrufo M, De Feo M, De Santo LS, et al. Risk of warfarin during pregnancy with mechanical valve prostheses. Obstet Gynecol. 2002;99:35-40.

5. Bates SM, Greer IA, Hirsh J, Ginsberg JS. Use of antithrombotic agents during pregnancy: the Seventh ACCP Conference on Antithrombotic and Thrombolytic Therapy. Chest. 2004;126(3 Suppl):627S-44S.

6. Ginsberg JS, Chan WS, Bates SM, Kaatz S. Anticoagulation of pregnant women with mechanical heart valves. Arch Intern Med 2003;163:694-8.

7. Chan WS, Anand S, Ginsberg JS. Anticoagulation of pregnant women with mechanical heart valves: a systematic review of the literature. Arch Intern Med. 2000;160:191-6.

8. Gouveia AMM, Ávila WS, Martins CR, et al. Sucesso da contracepção reversível em mulheres portadoras de cardiopatias. Arq Bras Cardiol. 2005;83(Suppl 1):45.

Correspondência:

Walkiria Samuel Ávila

Rua Martiniano de Carvalho, 864, cj 1107/08

Bela Vista

CEP 01321-000 - São Paulo, SP 